\title{
Electromagnetic radiation in a time-varying background medium
}

\author{
Neil V. Budko* \\ Laboratory of Electromagnetic Research, Faculty of Electrical Engineering, Mathematics and Computer Science, \\ Delft University of Technology, Mekelweg 4, 2628 CD Delft, The Netherlands
}

(Received 2 June 2009; published 12 November 2009)

\begin{abstract}
Analytical solutions are presented for the electromagnetic radiation by an arbitrary pulsed source into a homogeneous time-varying background medium. In the constant-impedance case an explicit radiation formula is obtained for the synchronous permittivity and permeability described by any positive function of time. As might be expected, such a medium introduces significant spectral shifts and spatiotemporal modulation, which are analyzed here for the linear and exponential time variations of the medium parameters. In the varyingimpedance case the solution is obtained for the fourth-order polynomial time dependence of the permittivity. In addition to the spectral shifts and modulation this spatially homogeneous medium scatters the field introducing causal echoes at the receiver location.
\end{abstract}

DOI: 10.1103/PhysRevA.80.053817

\section{INTRODUCTION}

One of the most difficult and least understood topics in science is the interaction between dynamic processes with separate physical origins and causes. Yet, such processes are very common in nature and engineering. For example, the electromagnetic field interacts with an independently controlled time-varying medium whenever we try to visualize or measure an ultrafast thermal, chemical, or mechanical transition. A linear time-varying medium is also a good approximation for a nonlinear medium with a strong pumping field and a weak probing signal. Potential applications include novel imaging algorithms for dynamic processes in timevarying physical systems undergoing mechanical displacement, deformation, phase transition, chemical reaction [1-7]; creation of ultrashort impulses and fast adaptive optical elements $[8,9]$; and investigation of transients in wired and wireless information networks with a time-varying load [10].

At present we are generally aware that the electromagnetic field evolution in a time-varying medium cannot be reduced to a sequence of snapshots corresponding to the instantaneous values of the medium parameters at various times. A plethora of very interesting and poorly understood transient effects is expected to be present. Some of those are cross modulation, velocity modulation, and parametric amplification of waves, e.g., dramatic pulse reshaping in attosecond optics and exponential growth of disturbances in networks. So far, however, we do not have any general theoretical picture of these phenomena and do not understand connections between them, and therefore do not yet realize their full technological potential. Most of the practical modeling is numerical and does not allow for in-depth analysis. A few available, mainly one-dimensional, analytical models [11-13] are often quasistationary, perturbative, restrict the type of the time variation of the system parameters and the source, and have little to say about the near-field effects. Practically nothing is known about the influence of nonautonomous systems on the coherence of the field. Threedimensional studies $[14,15]$ were mostly concerned with the

\footnotetext{
*n.v.budko@tudelft.nl
}

PACS number(s): 42.25.Bs, 03.50.De, 41.20.Jb

closed systems, such as electromagnetic cavities and waveguides with perfectly conducting walls. In general, the authors of nonperturbative studies agree that the time variation of the medium at a scale comparable to the time variation of the source results in a significant spatiotemporal modulation of the field. Recently there has been some progress in the theory of media periodically varying in time [16], where—as it turns out—one can apply the temporal analog of the Bloch theorem. The analysis shows that such time-varying media introduce band gaps in the wave-number (momentum) domain, as opposed to the frequency-domain (energy) band gaps produced by the spatially periodic media.

To understand the spatiotemporal modulation of the timedomain source by the general time-varying medium, one has to carry out a systematic theoretical analysis of the simplest nonautonomous electromagnetic system-a homogeneous isotropic time-varying background medium. This is the subject of the present paper, where we shall consider the realistic vectorial three-dimensional field and arbitrary time-varying sources. This study is intended to fill some of the gaps in our knowledge, e.g., about the near-field behavior, polarization effects, and few-cycle pulsed sources. In addition, it provides valuable analytical solutions that are useful for the verification of numerical codes. The mathematical techniques applied here are standard, albeit involved. First, using a straightforward change of variable we obtain a completely explicit analytical solution for the constant-impedance case, where the time variation of the permittivity and permeability is synchronous, but otherwise arbitrary (positive function of time). The more difficult time-varying impedance case is treated using an adaptation of the method originally proposed by Shvartsburg which allows one to derive an explicit solution for a polynomial time dependence of the permittivity.

\section{CONSTANT-IMPEDANCE CASE}

We begin with a relatively simple problem where the permittivity and permeability of the medium vary synchronously, i.e.,

$$
\varepsilon(t)=\varepsilon_{0} a(t), \quad \mu(t)=\mu_{0} a(t),
$$

so that the medium impedance remains constant at all times, $\varepsilon(t) / \mu(t)=\varepsilon_{0} / \mu_{0}=$ const. Consider the Maxwell equations 


$$
\begin{gathered}
-\boldsymbol{\nabla} \times \mathbf{H}(\mathbf{x}, t)+\partial_{t} \mathbf{D}(\mathbf{x}, t)=-\mathbf{J}(\mathbf{x}, t), \\
\boldsymbol{\nabla} \times \mathbf{E}(\mathbf{x}, t)+\partial_{t} \mathbf{B}(\mathbf{x}, t)=0,
\end{gathered}
$$

with the corresponding constitutive relations

$$
\mathbf{D}(\mathbf{x}, t)=\varepsilon_{0} a(t) \mathbf{E}(\mathbf{x}, t), \quad \mathbf{B}(\mathbf{x}, t)=\mu_{0} a(t) \mathbf{H}(\mathbf{x}, t) .
$$

Our goal is to obtain an explicit solution, called the radiation formula, which gives the fields in terms of the given source current density $\mathbf{J}$. Although the procedure is pretty standard, it will be presented in full detail to clarify the differences with the case of time-varying impedance discussed in the next section. First, we rewrite the Maxwell equations as

$$
\begin{gathered}
-\nabla \times \mathbf{B}(\mathbf{x}, t)+\mu_{0} a(t) \partial_{t} \mathbf{D}(\mathbf{x}, t)=-\mu_{0} a(t) \mathbf{J}(\mathbf{x}, t), \\
\boldsymbol{\nabla} \times \mathbf{D}(\mathbf{x}, t)+\varepsilon_{0} a(t) \partial_{t} \mathbf{B}(\mathbf{x}, t)=0 .
\end{gathered}
$$

Now, we introduce a new variable with the physical dimension of time,

$$
\tau=\int_{t_{0}}^{t} \frac{d t^{\prime}}{a\left(t^{\prime}\right)}
$$

where $t_{0}$ is the switch-on moment of the causal source $\mathbf{J}(\mathbf{x}, t)$, i.e., $\mathbf{J}(\mathbf{x}, t)=0\left(t<t_{0}\right)$. The original time $t$ will now be considered an implicit function $t(\tau)$ defined by the expression above. Taking into account that

$$
\frac{\partial}{\partial t}=\frac{d \tau}{d t} \frac{\partial}{\partial \tau}=\frac{1}{a(t)} \frac{\partial}{\partial \tau}
$$

and denoting

$$
\mathbf{J}_{1}(\mathbf{x}, \tau)=a(\tau) \mathbf{J}(\mathbf{x}, \tau),
$$

we rewrite the Maxwell equations in terms of the variable $\tau$ as

$$
\begin{gathered}
-\nabla \times \mathbf{B}(\mathbf{x}, \tau)+\mu_{0} \partial_{\tau} \mathbf{D}(\mathbf{x}, \tau)=-\mu_{0} \mathbf{J}_{1}(\mathbf{x}, \tau), \\
\boldsymbol{\nabla} \times \mathbf{D}(\mathbf{x}, \tau)+\varepsilon_{0} \partial_{\tau} \mathbf{B}(\mathbf{x}, \tau)=0,
\end{gathered}
$$

where the known functions $a(\tau)$ and $\mathbf{J}(\mathbf{x}, \tau)$ in the right-hand side must be understood as $a(t(\tau))$ and $\mathbf{J}(\mathbf{x}, t(\tau))$. The function $t(\tau)$ is the inverse mapping with respect to substitution (5). This inverse mapping exists and is one to one within an interval [to be determined for each particular $a(t)$ ], if $a\left(t^{\prime}\right)$ $>0, t_{0} \leq t^{\prime} \leq t$. Up to the variable change and the right-hand side, the Maxwell equations (8) are identical to the Maxwell equations in vacuum and have a well-known analytical solution. One usually starts by eliminating one of the unknowns, say $\mathbf{B}$, and arriving at the following second-order vector wave equation for $\mathbf{D}$ :

$$
\boldsymbol{\nabla} \times \boldsymbol{\nabla} \times \mathbf{D}(\mathbf{x}, \tau)+\frac{1}{c_{0}^{2}} \partial_{\tau}^{2} \mathbf{D}(\mathbf{x}, \tau)=-\frac{1}{c_{0}^{2}} \partial_{\tau} \mathbf{J}_{1}(\mathbf{x}, \tau),
$$

where $c_{0}=1 / \sqrt{\varepsilon_{0} \mu_{0}}$. From Eq. (8) we derive the compatibility relation

$$
\boldsymbol{\nabla} \cdot \mathbf{D}(\mathbf{x}, \tau)=-(\boldsymbol{\nabla} \cdot) \int_{0}^{\tau} \mathbf{J}_{1}\left(\mathbf{x}, \tau^{\prime}\right) d \tau^{\prime} .
$$

Rewriting Eq. (9) as

$$
\boldsymbol{\nabla}[\boldsymbol{\nabla} \cdot \mathbf{D}(\mathbf{x}, \tau)]-\Delta \mathbf{D}(\mathbf{x}, \tau)+\frac{1}{c_{0}^{2}} \partial_{\tau}^{2} \mathbf{D}(\mathbf{x}, \tau)=-\frac{1}{c_{0}^{2}} \partial_{\tau} \mathbf{J}_{1}(\mathbf{x}, \tau),
$$

and applying Eq. (10), we arrive at

$\Delta \mathbf{D}(\mathbf{x}, \tau)-\frac{1}{c_{0}^{2}} \partial_{\tau}^{2} \mathbf{D}(\mathbf{x}, \tau)=\frac{1}{c_{0}^{2}} \partial_{\tau} \mathbf{J}_{1}(\mathbf{x}, \tau)-\nabla(\nabla \cdot) \int_{0}^{\tau} \mathbf{J}_{1}\left(\mathbf{x}, \tau^{\prime}\right) d \tau^{\prime}$.

Performing the Laplace transform with respect to $\tau$ and the three-dimensional Fourier transform with respect to $\mathbf{x}$, we obtain the following algebraic equation in the $(\mathbf{k}, s)$ domain:

$$
-|\mathbf{k}|^{2} \tilde{\mathbf{D}}(\mathbf{k}, s)-\gamma_{0}^{2} \tilde{\mathbf{D}}(\mathbf{k}, s)=\frac{s}{c_{0}^{2}} \widetilde{\mathbf{J}}_{1}(\mathbf{k}, s)+\mathbf{k}(\mathbf{k} \cdot) \frac{1}{s} \widetilde{\mathbf{J}}_{1}(\mathbf{k}, s),
$$

where $\gamma_{0}=s \sqrt{\varepsilon_{0} \mu_{0}}=s / c_{0}$. This equation can be solved as

$$
\tilde{\mathbf{D}}(\mathbf{k}, s)=-\frac{1}{|\mathbf{k}|^{2}+\gamma_{0}^{2}}\left[\frac{s}{c_{0}^{2}} \tilde{\mathbf{J}}_{1}(\mathbf{k}, s)+\mathbf{k}(\mathbf{k} \cdot) \frac{1}{s} \tilde{\mathbf{J}}_{1}(\mathbf{k}, s)\right] .
$$

Carrying out the inverse three-dimensional Fourier transform we obtain the $(\mathbf{x}, s)$-domain solution as

$$
\begin{aligned}
\hat{\mathbf{D}}(\mathbf{x}, s)= & -\int_{\mathbf{x}^{\prime} \in \mathbb{R}^{3}} g\left(\mathbf{x}-\mathbf{x}^{\prime}, s\right) \frac{s}{c_{0}^{2}} \hat{\mathbf{J}}_{1}\left(\mathbf{x}^{\prime}, s\right) d \mathbf{x}^{\prime} \\
& +\nabla(\nabla \cdot) \int_{\mathbf{x}^{\prime} \in \mathbb{R}^{3}} g\left(\mathbf{x}-\mathbf{x}^{\prime}, s\right) \frac{1}{s} \hat{\mathbf{J}}_{1}\left(\mathbf{x}^{\prime}, s\right) d \mathbf{x}^{\prime},
\end{aligned}
$$

where the scalar Green's function is

$$
g(\mathbf{x}, s)=\frac{e^{-\gamma_{0}|\mathbf{x}|}}{4 \pi|\mathbf{x}|} .
$$

Computing the spatial derivatives and grouping the terms according to their spatial decay factors, we get

$$
\begin{aligned}
\hat{\mathbf{D}}(\mathbf{x}, s)= & \int_{\mathbf{x}^{\prime} \in \mathbb{R}^{3}} \frac{e^{-\gamma_{0}\left|\mathbf{x}-\mathbf{x}^{\prime}\right|}}{4 \pi\left|\mathbf{x}-\mathbf{x}^{\prime}\right|^{3}}[3 Q-I] \frac{1}{s} \hat{\mathbf{J}}_{1}\left(\mathbf{x}^{\prime}, s\right) d \mathbf{x}^{\prime} \\
& +\int_{\mathbf{x}^{\prime} \in \mathbb{R}^{3}} \frac{e^{-\gamma_{0}\left|\mathbf{x}-\mathbf{x}^{\prime}\right|}}{4 \pi\left|\mathbf{x}-\mathbf{x}^{\prime}\right|^{2}}[3 Q-\mathbb{Q}] \frac{1}{c_{0}} \hat{\mathbf{J}}_{1}\left(\mathbf{x}^{\prime}, s\right) d \mathbf{x}^{\prime} \\
& +\int_{\mathbf{x}^{\prime} \in \mathbb{R}^{3}} \frac{e^{-\gamma_{0}\left|\mathbf{x}-\mathbf{x}^{\prime}\right|}}{4 \pi\left|\mathbf{x}-\mathbf{x}^{\prime}\right|}[Q-\mathbb{I}] \frac{s}{c_{0}^{2}} \hat{\mathbf{J}}_{1}\left(\mathbf{x}^{\prime}, s\right) d \mathbf{x}^{\prime},
\end{aligned}
$$

where $Q=\Theta(\Theta \cdot)$ is a dyadic tensor constructed from the unit vector $\boldsymbol{\Theta}=\left(\mathbf{x}-\mathbf{x}^{\prime}\right) /\left|\mathbf{x}-\mathbf{x}^{\prime}\right|$ and $\mathbb{I}$ is the Kronecker identity tensor with components $\delta_{n m}=1(n=m), \delta_{n m}=0(n \neq m)$. Note that $Q$ is algebraically a projector, i.e., $Q^{2}=Q$. From the geometrical point of view it projects the source vector on the direction of observation $\boldsymbol{\Theta}$, i.e., it produces the "longitudi- 
nal" component of the field, whereas the complementary orthogonal projector $\mathbb{I}-\mathrm{Q}$ satisfies $[\mathbb{I}-\mathrm{Q}] \mathrm{Q}=0$ and projects the source vector on the direction orthogonal to $\boldsymbol{\Theta}$, i.e., it gives the "transverse" component. Thus, the far-field term in Eq. (17) is purely transverse as expected. The inverse Laplace transform of expressions (15) and (17) gives the $(\mathbf{x}, \tau)$-domain solution

$$
\begin{aligned}
\mathbf{E}(\mathbf{x}, \tau)= & \int_{\mathbf{x}^{\prime} \in \mathbb{R}^{3}} \frac{1}{4 \pi\left|\mathbf{x}-\mathbf{x}^{\prime}\right|^{3}}[3 Q-I] \int_{0}^{\tau_{\mathrm{r}}} \frac{a\left(\tau^{\prime}\right) \mathbf{J}\left(\mathbf{x}^{\prime}, \tau^{\prime}\right)}{\varepsilon_{0} a(\tau)} d \tau^{\prime} d \mathbf{x}^{\prime} \\
& +\int_{\mathbf{x}^{\prime} \in \mathbb{R}^{3}} \frac{1}{4 \pi\left|\mathbf{x}-\mathbf{x}^{\prime}\right|^{2}}[3 Q-\mathbb{I}] \frac{a\left(\tau_{\mathrm{r}}\right) \mathbf{J}\left(\mathbf{x}^{\prime}, \tau_{\mathrm{r}}\right)}{c_{0} \varepsilon_{0} a(\tau)} d \mathbf{x}^{\prime} \\
& +\int_{\mathbf{x}^{\prime} \in \mathbb{R}^{3}} \frac{1}{4 \pi\left|\mathbf{x}-\mathbf{x}^{\prime}\right|^{2}}[\mathrm{Q}-\mathbb{I}] \frac{\partial_{\pi}\left[a(\tau) \mathbf{J}\left(\mathbf{x}^{\prime}, \tau\right)\right]_{\tau=\tau_{\mathrm{r}}}}{c_{0}^{2} \varepsilon_{0} a(\tau)} d \mathbf{x}^{\prime},
\end{aligned}
$$

Where we have used $\mathbf{D}(\mathbf{x}, \tau)=\varepsilon(\tau) \mathbf{E}(\mathbf{x}, \tau)$ and Eq. (7). The retarded $\tau$ time is

$$
\tau_{\mathrm{r}}=\tau-\frac{\left|\mathbf{x}-\mathbf{x}^{\prime}\right|}{c_{0}} .
$$

The actual domain of integration while evaluating Eq. (18) will be reduced to $\mathbf{x}^{\prime} \in D_{\text {src }}$, where $D_{\text {src }}$ is the finite spatial domain occupied by the source, i.e., $\mathbf{J}\left(\mathbf{x}^{\prime}, \tau\right) \neq 0, \mathbf{x}^{\prime} \in D_{\text {src }}$. To obtain the $(\mathbf{x}, t)$-domain solution we now need to introduce the inverse $\tau$ function $t(\tau)$, which assigns a particular and unique proper time $t$, to any given $\tau$. If the medium is time invariant, then $t(\tau)=t, \mu(\tau)=\mu_{0}, \varepsilon(\tau)=\varepsilon_{0}$, reducing Eq. (18) to the well-known free-space radiation formula (see e.g., [17])

$$
\begin{aligned}
\mathbf{E}(\mathbf{x}, t)= & \int_{\mathbf{x}^{\prime} \in \mathrm{R}^{3}} \frac{1}{4 \pi\left|\mathbf{x}-\mathbf{x}^{\prime}\right|^{3}}[3 Q-\mathbb{I}] \int_{t_{0}}^{t_{\mathrm{r}}} \frac{\mathbf{J}\left(\mathbf{x}^{\prime}, t^{\prime}\right)}{\varepsilon_{0}} d t^{\prime} d \mathbf{x}^{\prime} \\
& +\int_{\mathbf{x}^{\prime} \in \mathrm{R}^{3}} \frac{1}{4 \pi\left|\mathbf{x}-\mathbf{x}^{\prime}\right|^{2}}[3 Q-I] \frac{\mathbf{J}\left(\mathbf{x}^{\prime}, t_{\mathrm{r}}\right)}{c_{0} \varepsilon_{0}} d \mathbf{x}^{\prime} \\
& +\int_{\mathbf{x}^{\prime} \in \mathbb{R}^{3}} \frac{1}{4 \pi\left|\mathbf{x}-\mathbf{x}^{\prime}\right|^{[}}[\mathrm{Q}-\mathbb{I}] \frac{\left[\partial_{t} \mathbf{J}\left(\mathbf{x}^{\prime}, t\right)\right]_{t=t_{\mathrm{r}}}}{c_{0}^{2} \varepsilon_{0}} d \mathbf{x}^{\prime},
\end{aligned}
$$

and the retarded time is $t_{\mathrm{r}}=t-\left|\mathbf{x}-\mathbf{x}^{\prime}\right| / c_{0}$. Comparing Eqs.
(18) and (20) we see that the difference between the timevarying and the time-invariant (constant-impedance) background media is in the presence of the time weighting and in the modulation of the source current density. It is interesting to note that, although the formally defined velocity of light in a time-varying medium would be also time-varying, i.e., $c$ $=1 / \sqrt{\varepsilon(t) \mu(t)}=c_{0} / a(t)$, the radiation formula (18) features the time delay factor $\left|\mathbf{x}-\mathbf{x}^{\prime}\right| / c_{0}$ [see Eq. (19)], containing the usual constant speed of light in vacuum. In other words, the propagation of light in a time-varying constant-impedance medium may be viewed as a propagation in vacuum where the causal time delay is measured in terms of the "universal" $\tau$ time. The actual measurements however are done in terms of the usual $t$ time. Hence, to translate the abstract $\tau$-domain picture into the $t$-domain reality, we need to find the inverse mapping $t(\tau)$ and use it in the given functions $a(t)$ and $\mathbf{J}(\mathbf{x}, t)$ thus obtaining the functions $a(\tau)$ and $\mathbf{J}(\mathbf{x}, \tau)$ appearing in the radiation formula (18). Then, after performing all the necessary mathematical operations with respect the $\tau$ variable in the right-hand side of Eq. (18), we shall try to recognize the $t$-domain solution.

Although, the inverse function $t(\tau)$ exists for any $a\left(t^{\prime}\right)$ $>0, t_{0} \leq t^{\prime} \leq t$, it can be found explicitly only if the integral in Eq. (5) can be evaluated analytically and the subsequent algebraic equation can be analytically solved for $t$. Consider a linear time dependence,

$$
a(t)=a_{0}+b t .
$$

Then, from Eq. (5) we find

$$
\begin{gathered}
t(\tau)=\frac{a_{0}+b t_{0}}{b} e^{b \tau}-\frac{a_{0}}{b}, \\
a(\tau)=\left(a_{0}+b t_{0}\right) e^{b \tau} .
\end{gathered}
$$

A time-harmonic source $\mathbf{J}(\mathbf{x}, t)=\mathbf{I}(\mathbf{x}) \cos (\omega t)$ will have the following representation in the $\tau$ domain:

$$
\mathbf{J}(\mathbf{x}, \tau)=\mathbf{I}(\mathbf{x}) \cos [\omega t(\tau)]=\mathbf{I}(\mathbf{x}) \cos \left[\omega\left(\frac{a_{0}+b t_{0}}{b} e^{b \tau}-\frac{a_{0}}{b}\right)\right] .
$$

In the far-field zone the electric field strength is proportional to the last term in Eq. (18). Explicitly we obtain

$$
\begin{aligned}
\mathbf{E}(\mathbf{x}, \tau) \approx & \int_{\mathbf{x}^{\prime} \in \mathbb{R}^{3}} \frac{[\mathrm{Q}-I] \mathbf{I}\left(\mathbf{x}^{\prime}\right) \partial_{\tau}\left\{\left(a_{0}+b t_{0}\right) e^{b \tau} \cos \left[\omega\left(\frac{a_{0}+b t_{0}}{b} e^{b \tau}-\frac{a_{0}}{b}\right)\right]\right\}}{4 \pi c_{0}^{2} \varepsilon_{0}\left|\mathbf{x}-\mathbf{x}^{\prime}\right|\left(a_{0}+b t_{0}\right) e^{b \tau}} d \mathbf{x}^{\prime} \\
= & \int_{\mathbf{x}^{\prime} \in \tau^{3}} \frac{[\mathrm{Q}-\mathbb{I}] \mathbf{I}\left(\mathbf{x}^{\prime}\right) b e^{b \tau_{\mathrm{r}}} \cos \left[\omega\left(\frac{a_{0}+b t_{0}}{b} e^{b \tau_{\mathrm{r}}}-\frac{a_{0}}{b}\right)\right]}{4 \pi c_{0}^{2} \varepsilon_{0}\left|\mathbf{x}-\mathbf{x}^{\prime}\right| e^{b \tau}} d \mathbf{x}^{\prime} \\
& +\int_{\mathbf{x}^{\prime} \in \mathbb{R}^{3}} \frac{[\mathrm{Q}-I] \mathbf{I}\left(\mathbf{x}^{\prime}\right) \omega\left(a_{0}+b t_{0}\right) e^{2 b \tau_{\mathrm{r}}} \sin \left[\omega\left(\frac{a_{0}+b t_{0}}{b} e^{b \tau_{\mathrm{r}}}-\frac{a_{0}}{b}\right)\right]}{4 \pi c_{0}^{2} \varepsilon_{0}\left|\mathbf{x}-\mathbf{x}^{\prime}\right| e^{b \tau}} d \mathbf{x}^{\prime}
\end{aligned}
$$




$$
\begin{aligned}
& =\int_{\mathbf{x}^{\prime} \in \mathbb{R}^{3}} \frac{[Q-I] \mathbf{I}\left(\mathbf{x}^{\prime}\right) b e^{-\left(b / c_{0}\right)\left|\mathbf{x}-\mathbf{x}^{\prime}\right|} \cos \left[\omega\left(\frac{a_{0}+b t_{0}}{b} e^{b \tau} e^{-\left(b / c_{0}\right)\left|\mathbf{x}-\mathbf{x}^{\prime}\right|}-\frac{a_{0}}{b}\right)\right]}{4 \pi c_{0}^{2} \varepsilon_{0}\left|\mathbf{x}-\mathbf{x}^{\prime}\right|} d \mathbf{x}^{\prime} \\
& \quad+\int_{\mathbf{x}^{\prime} \in \mathbb{R}^{3}} \frac{[Q-\mathbb{I}] \mathbf{I}\left(\mathbf{x}^{\prime}\right) \omega\left(a_{0}+b t_{0}\right) e^{b \tau} e^{-2\left(b / c_{0}\right)\left|\mathbf{x}-\mathbf{x}^{\prime}\right|} \sin \left[\omega\left(\frac{a_{0}+b t_{0}}{b} e^{b \tau} e^{-\left(b / c_{0}\right)\left|\mathbf{x}-\mathbf{x}^{\prime}\right|}-\frac{a_{0}}{b}\right)\right]}{4 \pi c_{0}^{2} \varepsilon_{0}\left|\mathbf{x}-\mathbf{x}^{\prime}\right|} d \mathbf{x}^{\prime},
\end{aligned}
$$

where, using Eqs. (22) and (23), we recognize the $t$-domain result as

$$
\begin{aligned}
\mathbf{E}(\mathbf{x}, t) \approx & \int_{\mathbf{x}^{\prime} \in \mathbb{R}^{3}} \frac{[Q-\mathbb{I}] \mathbf{I}\left(\mathbf{x}^{\prime}\right) b e^{-\left(b / c_{0}\right)\left|\mathbf{x}-\mathbf{x}^{\prime}\right|} \cos \left[\omega^{\prime} t+\frac{a_{0}}{b}\left(\omega^{\prime}-\omega\right)\right]}{4 \pi c_{0}^{2} \varepsilon_{0}\left|\mathbf{x}-\mathbf{x}^{\prime}\right|} d \mathbf{x}^{\prime} \\
& +\int_{\mathbf{x}^{\prime} \in \mathbb{R}^{3}} \frac{[Q-\mathbb{I}] \mathbf{I}\left(\mathbf{x}^{\prime}\right) \omega^{\prime} a(t) e^{-\left(b / c_{0}\right)\left|\mathbf{x}-\mathbf{x}^{\prime}\right|} \sin \left[\omega^{\prime} t+\frac{a_{0}}{b}\left(\omega^{\prime}-\omega\right)\right]}{4 \pi c_{0}^{2} \varepsilon_{0}\left|\mathbf{x}-\mathbf{x}^{\prime}\right|} d \mathbf{x}^{\prime} .
\end{aligned}
$$

In this formula we have a new frequency

$$
\omega^{\prime}=\omega e^{-\left(b / c_{0}\right)\left|\mathbf{x}-\mathbf{x}^{\prime}\right|},
$$

showing that one of the effects due to the time-varying background is a shift of the observed frequency depending on the distance between the source and the receiver. In particular, a medium varying linearly with time at a rate $b$ introduces a frequency shift varying exponentially with distance (27) blueshift for negative $b$ and redshift for the positive one. We also notice an additional exponential distance factor in (26)_-decay for redshift and amplification for blueshift. Tracing back to Eq. (18) we see that the latter parametric amplification is due to the modulation of the source by the $a(\tau)$ function, which produces other interesting phenomena as well. For example, if the time variation of the current is such that

$$
\partial_{\tau}[a(\tau) \mathbf{J}(\mathbf{x}, \tau)]=0, \quad \text { i.e., } a(\tau) \mathbf{J}(\mathbf{x}, \tau)=\text { const, }
$$

then the far-field term in Eq. (18) disappears, meaning that the field will die-off very rapidly with the distance from the source, and that the time-varying current-which would normally radiate-may be effectively silenced by a suitably time-varying homogeneous and lossless medium.

The other related but opposite phenomenon is the radiation by a dc-like current. Normally, a current which remains constant in time after it was switched on does not radiate into the far-field zone at later times (after the initial transient due to switch-on) neither does a charged particle moving at a constant velocity, whereas, in a time-varying medium, a constant current would produce a far-field radiation even at later times, with magnitude proportional to the magnitude of the current and with the time characteristics of $a(t)$. Indeed,

$$
\partial_{\tau}[a(\tau) \mathbf{J}(\mathbf{x})]=\mathbf{J}(\mathbf{x}) \partial_{\tau} a(\tau) \neq 0 .
$$

Obviously, it is the interaction of the quasistatic source and its quasistatic field with the time-varying medium, which is the true cause of the far-field radiation in this case. Despite this interaction no visible secondary sources will appear in the present constant-impedance case. An observer will perceive the spatial domain $D_{\text {src }}$, where $\mathbf{J}(\mathbf{x}) \neq 0$, as the only source of radiation.

All of this confirms that a time-varying medium, even a spatially homogeneous one, may produce a significant spacetime modulation of the electromagnetic field. Although a medium with constant impedance considered so far is a very rare thing, at least one physically interesting instant of such a medium is found in general relativity theory (GRT) $[18,19]$, where a linearly expanding universe corresponds to the linear $a(t)$ discussed above [apart for the current-modulating factor, which is $a^{4}(\tau)$ in GRT]. In particular, the application of the present analysis to the problem of Hubble's redshift law shows that a linearly expanding universe produces an exponential redshift, not a linear one as it is currently believed. In general, it may be shown, using only Eqs. (19) and (22), that any two temporal features of the time derivative of the modulated current density, say the zeros or extrema of the signal radiated by the source at times $t_{1}^{\mathrm{src}}$ and $t_{2}^{\mathrm{src}}$, with an interval $\Delta t^{\mathrm{src}}=t_{1}^{\mathrm{src}}-t_{2}^{\mathrm{src}}$ between them, will be detected by the receiver at times $t_{1}^{\mathrm{rec}}$ and $t_{2}^{\mathrm{rec}}$ with a different interval $\Delta t^{\mathrm{rec}}$ $=t_{1}^{\mathrm{rec}}-t_{2}^{\mathrm{rec}}$, namely,

$$
\frac{\Delta t^{\mathrm{rec}}}{\Delta t^{\mathrm{src}}}=e^{\left(b / c_{0}\right)\left|\mathbf{x}^{\mathrm{scc}}-\mathbf{x}^{\mathrm{rec}}\right|},
$$

where $\mathbf{x}^{\text {src }}$ and $\mathbf{x}^{\text {rec }}$ are the spatial locations of the source and the receiver, respectively. This is simply a generalization of the frequency shift law (27). In combination with the radiation formula (18) and the time-delay expression (19) this law provides an intuitively appealing picture of the expanding universe. First of all, there is no relative motion of the source and receiver, as the position vectors in the final radiation formula (18) are time independent. The speed of light also does not change as far as the $\tau$-domain picture is concerned. Yet, in the real $t$-domain world we observe the stretching of 
the time interval between certain characteristic events in the received signal according to Eq. (30).

Imagine two clocks, which are initially at the same location in space and are synchronized. Suppose that each second these clocks radiate a short impulse of light. Now, we place one of these clocks some distance away and observe that the interval between the impulses arriving from that clock is longer than one second (impulses have become broader in time too). We are naturally inclined to think that the remote clock started to walk slower for some reason and decide to go and check it. To our surprise, however, upon our arrival we find that everything is fine with this remote clock, and it is the other one which walks slower, judging from the impulses that are coming from it. We begin to suspect that there is a redshift involved due to the relative motion of the two clocks. Yet, when we put a ruler between them and observe the measured distance for some time, nothing seems to happen, and the clocks appear to be at rest with respect to each other. This, in short, is what happens in an expanding universe.

Due to its importance in cosmology, especially for understanding the process of inflation proposed within the big bang theory, we shall consider here the case of exponentially growing $a(t)$ as well. Suppose

$$
a(t)=a_{0} e^{b t} .
$$

Then, from Eq. (5), the inverse function $t(\tau)$ is found to be

$$
t(\tau)=-\frac{1}{b} \ln \left[e^{-b t_{0}}-a_{0} b \tau\right] .
$$

It is obvious that this inverse mapping is only defined for $\tau$ $<e^{-b t_{0}} /\left(a_{0} b\right)$ since otherwise the physical time $t$ would become complex. Therefore, according to Eq. (19), we may conclude that the light impulse radiated at $\tau$ will be confined to the spatial radius

$$
R=\frac{c_{0}}{a_{0} b} e^{-b t_{0}}-c_{0} \tau,
$$

effectively excluding any communication between points further than $R$ apart. Alternatively, one could speculate that the laws of light propagation are different beyond that radius, which is highly unlikely. Computing the ratio of time intervals between the corresponding features (e.g., zeros) of the electromagnetic signal at the source and receiver locations, we get the following expression:

$$
\frac{\Delta t^{\mathrm{rec}}}{\Delta t^{\mathrm{src}}}=\log _{s} r
$$

where

$$
\begin{gathered}
s=\frac{e^{-b t_{0}}-a_{0} b \tau_{2}^{\mathrm{src}}}{e^{-b t_{0}}-a_{0} b \tau_{1}^{\mathrm{src}}}, \\
r=\frac{e^{-b t_{0}}-a_{0} b\left(\tau_{2}^{\mathrm{src}}+\left|\mathbf{x}-\mathbf{x}^{\prime}\right| / c_{0}\right)}{e^{-b t_{0}}-a_{0} b\left(\tau_{1}^{\mathrm{src}}+\left|\mathbf{x}-\mathbf{x}^{\prime}\right| / c_{0}\right)} .
\end{gathered}
$$

This proves wrong the common assumption that an exponentially expanding universe introduces an exponential cosmo- logical redshift. The redshift appears also to depend on the interval between $\tau_{1}^{\mathrm{src}}$ and $\tau_{2}^{\mathrm{src}}$, i.e., it is inhomogeneousdifferent frequencies will have different shifts.

\section{TIME-VARYING IMPEDANCE}

The problem for a general time-varying impedance with asynchronously varying permittivity and permeability so far resists analytical treatment. However, for a class of timevarying impedances, where $\mu(t)$ is a constant and $\varepsilon(t)$ is a fourth-order polynomial in time, a useful analytical result can still be obtained. The solution can even be put in a form of a radiation formula similar to Eqs. (18) and (20) revealing important differences with the stationary medium and the constant-impedance cases. The technique is due to Shvartsburg [14], who considered mainly the source-free onedimensional Maxwell equations. Here, this method is extended to arbitrary pulsed sources in three dimensions. We denote

$$
\varepsilon(t)=\varepsilon_{0} a(t)=\varepsilon_{0} u^{2}(t), \quad \mu(t)=\mu_{0}=\text { const. }
$$

We again introduce a (slightly different) timelike variable

$$
\tau=\int_{t_{0}}^{t} \frac{d t^{\prime}}{\sqrt{a\left(t^{\prime}\right)}}=\int_{t_{0}}^{t} \frac{d t^{\prime}}{u\left(t^{\prime}\right)},
$$

so that the partial derivatives are related as

$$
\frac{\partial}{\partial t}=\frac{1}{u(t)} \frac{\partial}{\partial \tau}
$$

In addition to this variable change, an unknown function is introduced as

$$
\mathbf{F}(\mathbf{x}, t)=\frac{\mathbf{D}(\mathbf{x}, t)}{\sqrt{u(t)}} .
$$

To deal with the three-dimensional vector case some preliminary work needs to be done, consisting of deriving the $(\mathbf{x}, \tau)$-domain compatibility relation for the $\mathbf{F}$ field. It can be obtained from the $(\mathbf{x}, \tau)$-domain Maxwell equations and turns out to be

$$
\boldsymbol{\nabla} \cdot \mathbf{F}(\mathbf{x}, \tau)=-\frac{1}{\sqrt{u(\tau)}}(\boldsymbol{\nabla} \cdot) \int_{0}^{\tau} u\left(\tau^{\prime}\right) \mathbf{J}\left(\mathbf{x}, \tau^{\prime}\right) d \tau^{\prime}
$$

Now we go back to the $(\mathbf{x}, t)$-domain Maxwell equations and eliminate $\mathbf{H}$, thus obtaining the second-order vector wave equation for $\mathbf{D}$,

$$
\boldsymbol{\nabla} \times \boldsymbol{\nabla} \times \mathbf{D}(\mathbf{x}, t)+\frac{u^{2}(t)}{c_{0}^{2}} \partial_{t}^{2} \mathbf{D}(\mathbf{x}, t)=-\frac{u^{2}(t)}{c_{0}^{2}} \partial_{t} \mathbf{J}(\mathbf{x}, t) .
$$

Performing both the function and the variable change and using Eq. (40) we arrive at 


$$
\begin{gathered}
\Delta \mathbf{F}(\mathbf{x}, \tau)-\frac{1}{c_{0}^{2}} \partial_{\tau}^{2} \mathbf{F}(\mathbf{x}, \tau)-\frac{1}{c_{0}^{2}}\left[\frac{1}{2} u(t) \partial_{t}^{2} u(t)-\frac{1}{4}\left[\partial_{t} u(t)\right]^{2}\right] \mathbf{F}(\mathbf{x}, \tau) \\
=\frac{\sqrt{u(\tau)}}{c_{0}^{2}} \partial_{\tau} \mathbf{J}(\mathbf{x}, \tau)-\frac{1}{\sqrt{u(\tau)}} \boldsymbol{\nabla}(\boldsymbol{\nabla} \cdot) \int_{0}^{\tau} u\left(\tau^{\prime}\right) \mathbf{J}\left(\mathbf{x}, \tau^{\prime}\right) d \tau^{\prime} .
\end{gathered}
$$

Now, and this is the essence of the Shvartsburg method [14], we shall limit the time dependence of the medium, i.e., the class of functions $u(t)$, to those satisfying the nonlinear equation

$$
\frac{1}{2} u(t) \partial_{t}^{2} u(t)-\frac{1}{4}\left[\partial_{t} u(t)\right]^{2}=\frac{1}{T^{2}},
$$

where $T$ is a real constant with the physical dimension of time. In that case Eq. (41) reduces to

$$
\begin{aligned}
& \Delta \mathbf{F}(\mathbf{x}, \tau)-\frac{1}{c_{0}^{2}} \partial_{\tau}^{2} \mathbf{F}(\mathbf{x}, \tau)-\frac{1}{T^{2} c_{0}^{2}} \mathbf{F}(\mathbf{x}, \tau) \\
& =\frac{\sqrt{u(\tau)}}{c_{0}^{2}} \partial_{\tau} \mathbf{J}(\mathbf{x}, \tau)-\frac{1}{\sqrt{u(\tau)}} \boldsymbol{\nabla}(\boldsymbol{\nabla} \cdot) \int_{0}^{\tau} u\left(\tau^{\prime}\right) \mathbf{J}\left(\mathbf{x}, \tau^{\prime}\right) d \tau^{\prime},
\end{aligned}
$$

which can be solved analytically for any source. The functions $u(t)$ leading to this simplified equation are the solutions of Eq. (43) and have the form

$$
u(t)=a+b t+c t^{2} .
$$

This polynomial function allows one to model both rising and decaying profiles of $\varepsilon(t)=\varepsilon_{0} u^{2}(t)$, while the parameter $T=\left(c-b^{2} / 4\right)^{-1 / 2}$ determines the temporal scale of variations.

The solution of Eq. (44) is obtained via the same path as in the previous section. First, we transform the equation to the $(\mathbf{k}, s)$ domain and solve it there. Then, we carry out the inverse Fourier transform and obtain the following $(\mathbf{x}, s)$-domain solution:

$$
\begin{aligned}
\hat{\mathbf{F}}(\mathbf{x}, s)= & \int_{\mathbf{x}^{\prime} \in \mathbb{R}^{3}} \frac{e^{-\gamma\left|\mathbf{x}-\mathbf{x}^{\prime}\right|}}{4 \pi\left|\mathbf{x}-\mathbf{x}^{\prime}\right|^{3}}[3 Q-\mathbb{Q}] \hat{\mathbf{J}}_{1}\left(\mathbf{x}^{\prime}, s\right) d \mathbf{x}^{\prime} \\
& +\int_{\mathbf{x}^{\prime} \in \mathrm{R}^{3}} \frac{\gamma e^{-\gamma\left|\mathbf{x}-\mathbf{x}^{\prime}\right|}}{4 \pi\left|\mathbf{x}-\mathbf{x}^{\prime}\right|^{2}}[3 Q-\mathbb{I}] \hat{\mathbf{J}}_{1}\left(\mathbf{x}^{\prime}, s\right) d \mathbf{x}^{\prime} \\
& +\int_{\mathbf{x}^{\prime} \in \mathbb{R}^{3}} \frac{\gamma^{2} e^{-\gamma\left|\mathbf{x}-\mathbf{x}^{\prime}\right|}}{4 \pi\left|\mathbf{x}-\mathbf{x}^{\prime}\right|} \mathrm{Q} \hat{\mathbf{J}}_{1}\left(\mathbf{x}^{\prime}, s\right) d \mathbf{x}^{\prime} \\
& -\int_{\mathbf{x}^{\prime} \in \mathbb{R}^{3}} \frac{e^{-\gamma\left|\mathbf{x}-\mathbf{x}^{\prime}\right|}}{4 \pi\left|\mathbf{x}-\mathbf{x}^{\prime}\right|} \frac{1}{c_{0}^{2}} \hat{\mathbf{J}}_{2}\left(\mathbf{x}^{\prime}, s\right) d \mathbf{x}^{\prime},
\end{aligned}
$$

where the currents are the $(\mathbf{x}, s)$-domain images of

$$
\begin{gathered}
\mathbf{J}_{1}(\mathbf{x}, \tau)=\frac{1}{\sqrt{u}(\tau)} \int_{0}^{\tau} u\left(\tau^{\prime}\right) \mathbf{J}\left(\mathbf{x}, \tau^{\prime}\right) d \tau^{\prime}, \\
\mathbf{J}_{2}(\mathbf{x}, \tau)=\sqrt{u(\tau)} \partial_{\tau} \mathbf{J}(\mathbf{x}, \tau) .
\end{gathered}
$$

The difference with Eq. (17) is mainly in the mathematical form of the parameter $\gamma$, which is now

$$
\gamma=\frac{1}{c_{0}} \sqrt{s^{2}+1 / T^{2}} .
$$

Obviously, for $1 / T^{2}=0$, corresponding to the infinitely slow variation of $\varepsilon(t)$, we recover the free-space case $\gamma=s / c_{0}$. To transform the result to the $(\mathbf{x}, \tau)$ domain, we recall the following known Laplace transform pairs:

$$
\begin{aligned}
e^{-k \sqrt{s^{2}+a^{2}}}, \quad(k>0) \rightarrow \delta(t-k)-\frac{a k}{\sqrt{t^{2}-k^{2}}} J_{1}\left(a \sqrt{t^{2}-k^{2}}\right) \\
\times H(t-k), \\
\frac{e^{-k \sqrt{s^{2}+a^{2}}}}{\sqrt{s^{2}+a^{2}}}, \quad(k \geq 0) \rightarrow J_{0}\left(a \sqrt{t^{2}-k^{2}}\right) H(t-k),
\end{aligned}
$$

where $H(t-k)$ is a unit step function located at $t=k$. Using these transforms we can write the result as

$$
\begin{aligned}
\mathbf{F}(\mathbf{x}, \tau)= & \int_{\mathbf{x}^{\prime} \in \mathbb{R}^{3}} \frac{1}{4 \pi\left|\mathbf{x}-\mathbf{x}^{\prime}\right|^{3}}[3 Q-I] \mathbf{J}_{1}\left(\mathbf{x}^{\prime}, \tau_{\mathrm{r}}\right) d \mathbf{x}^{\prime}-\frac{1}{c_{0} T} \int_{\mathbf{x}^{\prime} \in \mathbb{R}^{3}} \frac{1}{4 \pi\left|\mathbf{x}-\mathbf{x}^{\prime}\right|^{2}}[3 Q-I] \int_{0}^{\tau} g_{1}\left(\mathbf{x}-\mathbf{x}^{\prime}, \tau-\tau^{\prime}\right) \mathbf{J}_{1}\left(\mathbf{x}^{\prime}, \tau^{\prime}\right) d \tau^{\prime} d \mathbf{x}^{\prime} \\
& +\frac{1}{c_{0}} \int_{\mathbf{x}^{\prime} \in \mathbb{R}^{3}} \frac{1}{4 \pi\left|\mathbf{x}-\mathbf{x}^{\prime}\right|^{2}}[3 Q-\mathbb{I}] \int_{0}^{\tau} g_{0}\left(\mathbf{x}-\mathbf{x}^{\prime}, \tau-\tau^{\prime}\right)\left[\partial_{\tau^{\prime}}^{2}+\frac{1}{T^{2}}\right] \mathbf{J}_{1}\left(\mathbf{x}^{\prime}, \tau^{\prime}\right) d \tau^{\prime} d \mathbf{x}^{\prime} \\
& +\frac{1}{c_{0}^{2}} \int_{\mathbf{x}^{\prime} \in \mathbb{R}^{3}} \frac{1}{4 \pi\left|\mathbf{x}-\mathbf{x}^{\prime}\right|} Q\left[\partial_{\tau}^{2}+\frac{1}{T^{2}}\right] \mathbf{J}_{1}\left(\mathbf{x}^{\prime}, \tau_{\mathrm{r}}\right) d \mathbf{x}^{\prime}-\frac{1}{c_{0}^{3} T} \int_{\mathbf{x}^{\prime} \in \mathbb{R}^{3}} \frac{1}{4 \pi} Q \int_{0}^{\tau} g_{1}\left(\mathbf{x}-\mathbf{x}^{\prime}, \tau-\tau^{\prime}\right)\left[\partial_{\tau^{\prime}}^{2}+\frac{1}{T^{2}}\right] \mathbf{J}_{1}\left(\mathbf{x}^{\prime}, \tau^{\prime}\right) d \tau^{\prime} d \mathbf{x}^{\prime} \\
& -\frac{1}{c_{0}^{2}} \int_{\mathbf{x}^{\prime} \in \mathbb{R}^{3}} \frac{1}{4 \pi\left|\mathbf{x}-\mathbf{x}^{\prime}\right|} \mathbf{J}_{2}\left(\mathbf{x}^{\prime}, \tau_{\mathrm{r}}\right) d \mathbf{x}^{\prime}+\frac{1}{c_{0}^{3} T} \int_{\mathbf{x}^{\prime} \in \mathbb{R}^{3}} \frac{1}{4 \pi} \int_{0}^{\tau} g_{1}\left(\mathbf{x}-\mathbf{x}^{\prime}, \tau-\tau^{\prime}\right) \mathbf{J}_{2}\left(\mathbf{x}^{\prime}, \tau^{\prime}\right) d \tau^{\prime} d \mathbf{x}^{\prime},
\end{aligned}
$$

where 


$$
g_{0}(\mathbf{x}, \tau)=J_{0}\left(\frac{1}{T} \sqrt{\tau^{2}-\frac{|\mathbf{x}|^{2}}{c_{0}^{2}}}\right) H\left(\tau-\frac{|\mathbf{x}|}{c_{0}}\right), \quad g_{1}(\mathbf{x}, \tau)=\frac{J_{1}\left(\frac{1}{T} \sqrt{\tau^{2}-\frac{|\mathbf{x}|^{2}}{c_{0}^{2}}}\right)}{\sqrt{\tau^{2}-\frac{|\mathbf{x}|^{2}}{c_{0}^{2}}}} H\left(\tau-\frac{|\mathbf{x}|}{c_{0}}\right),
$$

and the retarded time is again given by Eq. (19). Notice that this retarded time appears both explicitly in some terms in Eq. (51) as well as implicitly in the Green's functions as a unit step multiplier; thus, the causality in the $(\mathbf{x}, \tau)$ domain is fully preserved. Computing out the time derivatives,

$$
\left[\partial_{\tau}^{2}+\frac{1}{T^{2}}\right] \mathbf{J}_{1}(\mathbf{x}, \tau)=v(\tau) \int_{0}^{\tau} u\left(\tau^{\prime}\right) \mathbf{J}\left(\mathbf{x}, \tau^{\prime}\right) d \tau^{\prime}+u^{1 / 2} \partial_{\tau} \mathbf{J}(\mathbf{x}, \tau), \quad v(\tau)=\frac{1}{T^{2} u^{1 / 2}}+\frac{3 \partial_{\tau} u}{4 u^{5 / 2}}-\frac{\partial_{\tau}^{2} u}{2 u^{3 / 2}},
$$

we can explicitly show all the terms in Eq. (51) as

$$
\begin{aligned}
\mathbf{E}(\mathbf{x}, \tau)= & \int_{\mathbf{x}^{\prime} \in \mathbb{R}^{3}} \frac{3 \mathrm{Q}-\mathbb{I}}{4 \pi\left|\mathbf{x}-\mathbf{x}^{\prime}\right|^{3}} \frac{1}{\varepsilon(\tau)} \int_{0}^{\tau_{\mathrm{r}}} \frac{u^{1 / 2}(\tau) u\left(\tau^{\prime}\right)}{u^{1 / 2}\left(\tau_{\mathrm{r}}\right)} \mathbf{J}\left(\mathbf{x}^{\prime}, \tau^{\prime}\right) d \tau^{\prime} d \mathbf{x}^{\prime} \\
& -\int_{\mathbf{x}^{\prime} \in \mathbb{R}^{3}} \frac{3 Q-I}{4 \pi\left|\mathbf{x}-\mathbf{x}^{\prime}\right|^{2}} \frac{1}{c_{0} \varepsilon(\tau)} \int_{0}^{\tau} g_{1}\left(\mathbf{x}-\mathbf{x}^{\prime}, \tau-\tau^{\prime}\right) \int_{0}^{\tau^{\prime}} \frac{u^{1 / 2}(\tau) u\left(\tau^{\prime \prime}\right)}{T u^{1 / 2}\left(\tau^{\prime}\right)} \mathbf{J}\left(\mathbf{x}^{\prime}, \tau^{\prime \prime}\right) d \tau^{\prime \prime} d \tau^{\prime} d \mathbf{x}^{\prime} \\
& +\int_{\mathbf{x}^{\prime} \in \mathbb{R}^{3}} \frac{3 Q-\mathbb{I}}{4 \pi\left|\mathbf{x}-\mathbf{x}^{\prime}\right|^{2}} \frac{1}{c_{0} \varepsilon(\tau)} \int_{0}^{\tau} g_{0}\left(\mathbf{x}-\mathbf{x}^{\prime}, \tau-\tau^{\prime}\right) \int_{0}^{\tau^{\prime}} u^{1 / 2}(\tau) v\left(\tau^{\prime}\right) u\left(\tau^{\prime \prime}\right) \mathbf{J}\left(\mathbf{x}^{\prime}, \tau^{\prime \prime}\right) d \tau^{\prime \prime} d \tau^{\prime} d \mathbf{x}^{\prime} \\
& +\int_{\mathbf{x}^{\prime} \in \mathbb{R}^{3}} \frac{3 Q-\mathbb{I}}{4 \pi\left|\mathbf{x}-\mathbf{x}^{\prime}\right|^{2}} \frac{1}{c_{0} \varepsilon(\tau)} \int_{0}^{\tau} g_{0}\left(\mathbf{x}-\mathbf{x}^{\prime}, \tau-\tau^{\prime}\right) u^{1 / 2}(\tau) u^{1 / 2}\left(\tau^{\prime}\right) \partial_{\tau^{\prime}} \mathbf{J}\left(\mathbf{x}^{\prime}, \tau^{\prime \prime}\right) d \tau^{\prime} d \mathbf{x}^{\prime} \\
& +\int_{\mathbf{x}^{\prime} \in \mathbb{R}^{3}} \frac{\mathrm{Q}}{4 \pi\left|\mathbf{x}-\mathbf{x}^{\prime}\right|} \frac{1}{c_{0}^{2} \varepsilon(\tau)} \int_{0}^{\tau_{\mathrm{r}}} u^{1 / 2}(\tau) v\left(\tau_{\mathrm{r}}\right) u\left(\tau^{\prime}\right) \mathbf{J}\left(\mathbf{x}^{\prime}, \tau^{\prime}\right) d \tau^{\prime} d \mathbf{x}^{\prime} \\
& -\int_{\mathbf{x}^{\prime} \in \mathbb{R}^{3}} \frac{\mathrm{Q}}{4 \pi} \frac{1}{c_{0}^{2} \varepsilon(\tau)} \int_{0}^{\tau} g_{1}\left(\mathbf{x}-\mathbf{x}^{\prime}, \tau-\tau^{\prime}\right) \int_{0}^{\tau^{\prime}} \frac{u^{1 / 2}(\tau) v\left(\tau^{\prime}\right) u\left(\tau^{\prime \prime}\right)}{c_{0} T} \mathbf{J}\left(\mathbf{x}^{\prime}, \tau^{\prime \prime}\right) d \tau^{\prime \prime} d \tau^{\prime} d \mathbf{x}^{\prime} \\
& -\int_{\mathbf{x}^{\prime} \in \mathbb{R}^{3}} \frac{\mathrm{Q}}{4 \pi} \frac{1}{c_{0}^{2} \varepsilon(\tau)} \int_{0}^{\tau} g_{1}\left(\mathbf{x}-\mathbf{x}^{\prime}, \tau-\tau^{\prime}\right) \frac{u^{1 / 2}(\tau) u^{1 / 2}\left(\tau^{\prime}\right)}{c_{0} T} \partial_{\tau^{\prime}} \mathbf{J}\left(\mathbf{x}^{\prime}, \tau^{\prime}\right) d \tau^{\prime} d \mathbf{x}^{\prime} \\
& +\int_{\mathbf{x}^{\prime} \in \mathbb{R}^{3}} \frac{Q}{4 \pi\left|\mathbf{x}-\mathbf{x}^{\prime}\right|} \frac{1}{c_{0}^{2} \varepsilon(\tau)} u^{1 / 2}(\tau) u^{1 / 2}\left(\tau_{\mathrm{r}}\right) \partial_{\tau} \mathbf{J}\left(\mathbf{x}^{\prime}, \tau_{\mathrm{r}}\right) d \mathbf{x}^{\prime} .
\end{aligned}
$$

There are some similarities here with the constant-impedance case considered in the previous section. First of all we see both the time weighting and the modulation of the source. However, there are also obvious differences. The time weighting (37) contains now the square root of $a(t)$. Hence, to achieve the exponential spectral shift (27) we need $u(t)$ $=a_{0}+b t$, i.e., a quadratic variation of $\varepsilon(t)$,

$$
\varepsilon(t)=\varepsilon_{0}\left(a_{0}+b t\right)^{2} .
$$

Further, there are terms containing spatial and $\tau$ convolutions with the Green's functions (52). These terms represent causal (since $\tau$ integration starts only after the wave front has reached the observation point) echoes due to the scattering of the field on the temporal variation of the medium impedance. Moreover, this echoes will be perceived by an observer as arriving from the spatial domains outside the original source domain $D_{\text {src }}$, where $\mathbf{J}(\mathbf{x}, t) \neq 0$. Physically, they will be pro- duced by the gradually expanding zone of the field-medium interaction, as follows from the arguments of the Green's functions (52).

Finally, we notice some additional longitudinal contributions in the far-field zone-last four terms in Eq. (54). Normally, the far-field contribution is purely transverse, i.e., it contains only one term with Q-I [see Eq. (20)], whereas here we have three additional terms with Q decaying at the typical far-field $\left|\mathbf{x}-\mathbf{x}^{\prime}\right|^{-1}$ rate. Of course, two of them are medium-induced echoes. However, one is definitely produced by the source alone. This means that the time-varying background could be used to propagate the longitudinal nearfield features of the source into the far field.

\section{CONCLUSIONS}

We considered the effects of two types of homogeneous time-varying background media on the electromagnetic field 
radiated by an arbitrary source. The first type is a medium with synchronously varying permittivity and permeability, where the impedance stays constant at all times. Such a medium describes the time-varying spatially flat gravitational background, e.g., the expanding universe, but it can also be used as an approximation for a metamaterial with the timevarying effective permittivity and permeability. The obtained analytical solution shows that under a change of time variable the problem is similar to the radiation in a stationary background medium and that the spatial location and extent of the source will be perceived by the observer as if the medium was stationary. Yet, the scaled time variable and the modulation of the source term produce significant changes in the space-time evolution of the field. We have shown that a linear variation of the medium with time produces a "cosmological" frequency shift, which is an exponential function of the distance between the source and the observer as well as an additional exponential factor affecting the amplitude of the received signal. We have also derived the "logarithmic" inhomogeneous frequency shift for a universe expanding exponentially in time.

The more down-to-earth case of the constant permeability and time-varying permittivity appeared, in fact, to be more difficult and was treated using the Shvartsburg method, which involves the change of the time variable as well as the change of the unknown. This method gives an analytical solution for a limited but useful class of time-varying permittivities. The obtained solution shows, in particular, that the medium with a quadratic temporal change in permittivity would also produce an exponential distance-dependent shift in the observed frequency. In addition, the received signal is shown to contain causal echoes induced by the scattering of waves on the time variations of the impedance. The spatial extent of the effective source perceived by the observer will grow as the field-medium interaction zone expands, and the field will no longer be purely transverse in the far-field zone. The modulation of the source, which occurs in both constant and time-varying impedance cases, may be used either for silencing or for amplification of certain sources. Surprisingly, we find that, in a time-varying background, a dc-like constant current radiates into the far-field zone even at later times (after the early-time transients), whereas a specific time-varying current does not.

\section{ACKNOWLEDGMENTS}

The author is grateful to Dr. B.-J. Kooij (Delft University of Technology) for pointing out some useful Laplace transform pairs and to Professor A. T. de Hoop (Delft University of Technology) for many fruitful discussions.
[1] L. Melton, Nature (London) 437, 775 (2005)

[2] D. D. Steele, T. L. Chenevert, A. R. Skovoroda, and S. Y. Emelianov, Phys. Med. Biol. 45, 1633 (2000).

[3] M. Bauer, J. Phys. D: Appl. Phys. 38, R253 (2005).

[4] I. Hjelte, M. N. Piancastelli, R. F. Fink, O. Björneholm, M. Bässler, R. Feifel, A. Giertz, H. Wang, K. Wiesner, A. Ausmees, C. Miron, S. L. Sorensen, and S. Svensson, Chem. Phys. Lett. 334, 151 (2001).

[5] A. J. Sederman, M. D. Mantle, and L. F. Gladden, J. Magn. Reson. 161, 15, (2003).

[6] T. Y. Choi, D. J. Hwang, and C. P. Grigoropoulos, Opt. Eng. (Bellingham) 42, 3383 (2003).

[7] L. Guérin, E. Collet, M.-H. Lemée-Cailleau, M. Buron-Le Cointe, H. Cailleau, A. Plech, M. Wulff, S.-Y. Koshihara, and T. Luty, Chem. Phys. 299, 163 (2004).

[8] D. M. Marom, D. Panasenko, P.-C. Sun, and Yeshaiahu Fainman, Opt. Lett. 24, 563 (1999).
[9] A. G. Davies, E. H. Linfield, and M. B. Johnston, Phys. Med. Biol. 47, 3679 (2002).

[10] R. P. Broadwater, A. H. Khan, H. E. Shaalan, and R. E. Lee, IEEE Trans. Power Deliv. 8, 294 (1993).

[11] K. S. Shifrin and I. G. Zolotov, Appl. Opt. 33, 7798 (1994).

[12] A. G. Nerukh, P. Sewell, and T. M. Benson, J. Lightwave Technol. 22, 1408 (2004).

[13] M. I. Bakunov and A. V. Maslov, Phys. Rev. Lett. 79, 4585 (1997).

[14] A. B. Shvartsburg, Phys. Usp. 48, 797 (2005).

[15] S. Aksoy and O. A. Tretyakov, J. Electromagn. Waves Appl. 16, 1535 (2002).

[16] J. R. Zurita-Sánchez, P. Halevi, and J. C. Cervantes-González, Phys. Rev. A 79, 053821 (2009).

[17] N. V. Budko, Phys. Rev. Lett. 102, 020401 (2009).

[18] U. Leonhardt and T. G. Philbin, New J. Phys. 8, 247 (2006).

[19] N. Budko, e-print arXiv:0904.3280. 